\title{
The Frequent Adiponutrin (PNPLA3) Variant p.Ile148Met Is Associated with Early Liver Injury: Analysis of a German Pediatric Cohort
}

\author{
Marcin Krawczyk, ${ }^{1,2}$ Roman Liebe, ${ }^{1,3}$ Ina B. Maier, ${ }^{4}$ Anna Janina Engstler, ${ }^{5}$ \\ Frank Lammert, ${ }^{1}$ and Ina Bergheim ${ }^{5}$ \\ ${ }^{1}$ Department of Medicine II, Saarland University Medical Center, Saarland University, 66421 Homburg, Germany \\ ${ }^{2}$ Laboratory of Metabolic Liver Diseases, Department of General, Transplant and Liver Surgery, Medical University of Warsaw, \\ 02-091 Warsaw, Poland \\ ${ }^{3}$ Klinik für Innere Medizin II, Klinikum Mannheim, Universität Heidelberg, 68135 Mannheim, Germany \\ ${ }^{4}$ Department of Nutritional Medicine, Universität Hohenheim, 70599 Stuttgart, Germany \\ ${ }^{5}$ Department of Nutritional Sciences, SD Model Systems of Molecular Nutrition, Friedrich-Schiller-Universität, 07743 Jena, Germany
}

Correspondence should be addressed to Marcin Krawczyk; marcin.krawczyk@uks.eu

Received 16 October 2014; Revised 5 March 2015; Accepted 5 March 2015

Academic Editor: Antoni Castells

Copyright (C) 2015 Marcin Krawczyk et al. This is an open access article distributed under the Creative Commons Attribution License, which permits unrestricted use, distribution, and reproduction in any medium, provided the original work is properly cited.

\begin{abstract}
Introduction. The common adiponutrin (PNPLA3) variant p.Ile148Met is associated with liver injury. Here, we investigate the association of this polymorphism with hepatic and metabolic traits in a pediatric cohort. Patients and Methods. The study cohort comprised 142 German children (age 5-9 years, 98 overweight, 19 children with NAFLD). Results. Overweight children presented with increased serum ALT $(P=0.001)$ and GGT $(P<0.001)$ activities. ALT activities differed significantly $(P=0.02)$ between carriers of different PNPLA3 genotypes in the entire study cohort, in normal weight children $(P=0.02)$ and in children younger than 7 years $(P=0.02)$. Carriers of the prosteatotic PNPLA3 genotype p.148Met/Met displayed higher ALT activities as compared to children with the frequent genotype p.148Ile/Ile $(P=0.01)$. The BMI was however a stronger predictor of ALT activities compared to the PNPLA3 genotype $(P<0.001$ and $P=0.06$, resp.). The variant was associated with increased serum glucose levels $(P=0.01)$ and HOMA index $(P=0.02)$ in carriers of the p.148Ile/Met genotype but did not affect other metabolic traits or the presence of NAFLD. Discussion. The frequent PNPLA3 variant p.Ile148Met is associated with serum ALT activities already at a young age.
\end{abstract}

\section{Introduction}

The global obesity epidemic has turned nonalcoholic fatty liver disease (NAFLD) from a relatively rare phenomenon to a global health problem [1]. While steatosis, the presence of lipid droplets, is itself not necessarily harmful, it can progress into nonalcoholic steatohepatitis (NASH) in both children [2] and adult patients [3]. The distinguishing factor between simple steatosis/NAFLD and NASH is hepatocyte injury, characterized by ballooning, apoptosis, and infiltrating inflammatory cells. Between 10 and $30 \%$ of individuals with NASH may develop cirrhosis, that is, functional loss of liver tissue due to excess deposition of extracellular matrix, within 10 years. The prevalence of pediatric NALFD has been estimated as high as $10 \%$ [4], and the frequency of NAFLD in overweight and obese children may reach $25 \%$ and $76 \%$, respectively [1]. However, as in many countries like Germany, assessment of liver status is not mandatory in children and adolescents and most available data to date were derived in clinical setting, the real figure may be even higher.

A nonsynonymous sequence variant (rs738409) of the human patatin-like phospholipase domain containing gene 3 (PNPLA3) resulting in the amino acid substitution p.Ile148Met has been identified as major determinant of increased liver fat content and key susceptibility factor for NAFLD in adults [5]. There have now been several studies 
TABLE 1: Clinical characteristics of the pediatric cohort.

\begin{tabular}{lc}
\hline Variables & Subject characteristics \\
\hline$N$ (male/female) & $142(64 / 78)$ \\
Age (years) & $7(5-9)^{\&}$ \\
Patients with NAFLD ${ }^{*}(N)$ & 19 \\
Overweight individuals $(N)$ & 98 \\
BMI-SDS & $1.56(-1.09-3.46)^{\&}$ \\
\hline
\end{tabular}

$\overline{{ }^{*} \text { Values are given as medians and ranges. }{ }^{*} \text { Detected by abdominal ultra- }}$ sonography.

BMI-SDS: body mass index-standard deviation score; NAFLD: nonalcoholic fatty liver disease.

in pediatric populations regarding the impact of rs738409 on the prevalence and progression of nonalcoholic fatty liver in childhood [6-8]. To test for an association of the PNPLA3 rs738409 variant with liver damage in very young children, we analyzed a pediatric cohort of 142 patients aged between 5 and 9 years (described in detail in [9]) for the impact of this genetic risk factor on subclinical liver damage and NAFLD. Furthermore, we investigated the impact of this polymorphism on metabolic and anthropometric traits.

\section{Patients and Methods}

The cohort analysed in the current study comprises 142 German children (100 Caucasians, 42 Asians, 64 boys, median age 7 years, age range 5-9 years, and median standard deviation score $(\mathrm{SDS})$-body mass index $(\mathrm{BMI})=1.56)$. The participants of the study were volunteers recruited between April 2009 and December 2010 from elementary and primary schools in the greater area of Stuttgart. In addition, information leaflets asking for volunteers were distributed at children's parties and family venues and in sports clubs.

Table 1 summarizes the characteristics of the studied cohort. All pediatric patients underwent careful clinical examination including measurements of anthropometric traits. The BMI values were stratified according to reference data for German children as follows: normal weight: BMI $<90$ percentile $\mathrm{BMI}$ and overweight: $\mathrm{BMI}>90$ percentile BMI [10]. Children with known viral and nonviral liver diseases, other than NAFLD, were excluded from the study. Moreover, children included had no known history of renal insufficiency, diabetes types 1 and 2, chronic disease of the gastrointestinal tract, or taking lipid-lowering drugs or drugs affecting lipid metabolism. NAFLD was assessed by abdominal ultrasound, which was performed in all study participants by experienced pediatricians. Presence of steatosis was graded according to the following scale: grade 0 : no steatosis, grade 1: mild steatosis, grade 2: moderate steatosis, and grade 3: severe steatosis [11]. In each patient we measured anthropometric parameters, and blood samples were obtained from fasted subjects. Liver functions tests as well as serum insulin levels were determined by standard clinical-chemical assays. Levels of concurrent fasting insulin and glucose were used to estimate insulin resistance using the homeostasis model assessment (IR-HOMA) index with the following formula: insulin $(\mathrm{mU} / \mathrm{mL}) \times$ fasting plasma glucose $(\mathrm{mmol} / \mathrm{L}) / 22.5$ [12]. Informed consent was obtained from at least one parent of each patient. The protocol of the study was approved by the Ethics Committee of the Landesärztekammer Baden-Württemberg, Stuttgart.

\section{Genotyping and Statistics}

In all individuals we genotyped the frequent PNPLA3 variant rs738409 according to the methodology described previously [13]. Briefly, the DNeasy Blood and Tissue Kit (Qiagen) was used to isolate genomic DNA from EDTA anticoagulated blood. PCR reactions contained $20 \mathrm{ng}$ DNA, $900 \mathrm{nM}$ of each primer, $1 \times$ TaqMan Universal Master Mix, and $200 \mathrm{nM}$ of VIC-labelled and FAM-labelled probes in $25 \mu \mathrm{L}$ reactions. Following amplification conditions were set $95^{\circ} \mathrm{C}$ for $10 \mathrm{~min}$, 40 cycles of $92^{\circ} \mathrm{C}$ for $15 \mathrm{~s}$, and $60^{\circ} \mathrm{C}$ for $1 \mathrm{~min}$. The quality was ensured by inclusion of negative controls and DNA samples with known PNPLA3 genotypes as internal controls. Finally, the genotyping results were analysed with the SDS software (version 2.0.5). Consistency of genotyping results with Hardy-Weinberg equilibrium (HWE) was tested with an exact test (http://ihg.gsf.de/cgi-bin/hw/hwal.pl). Association (case-control) analysis was performed in contingency tables (genotypes: Armitage's trend test; alleles: chi ${ }^{2}$ test). Kolmogorov-Smirnov's test was used to determine whether quantitative data had a normal distribution. The nonparametric analysis of variance (ANOVA) with post hoc tests was used to assess the difference in serum levels of liver enzymes and metabolic and anthropometric traits among carriers of different PNPLA3 genotypes. Regression analyses were performed to analyse effects of the PNPLA3 genotypes and BMI on increased serum ALT. Two-sided $P<0.05$ was considered significant. All tests were performed with GraphPad Prism 5.0 (GraphPad Software, San Diego, USA) or SPSS 20.0 (SPSS, Munich, Germany).

\section{Results}

Overall, 44 children presented with normal body weight (23 boys, age range $5-9$ years, median age 7 years) and 98 children were overweight (41 boys, age range 5-9 years, median age 7 years). As shown in Figures 1(a) and 1(b), obese children presented with significantly increased serum ALT and GGT activities $(P=0.001$ and $P<0.001$, resp.). On the other hand, we did not detect any differences in serum AST between both groups and there were no significant differences in age and gender distribution (all $P>0.05$ ). In the whole cohort we observed the following genotype frequencies: PNPLA3 p.148Ile/Ile $=75$ (52.8\%), PNPLA3 p.148Ile/Met $=$ 57 (40.1\%), and PNPLA3 p.148Met/Met $=10$ (7.1\%). These frequencies did not differ significantly from values presented in previous publications [14] and were in line with HWE $(P=0.99)$. Overall, there was no association between the PNPLA3 genotype and presence of fatty liver as assessed by abdominal sonography $(N=19, P>0.05)$. As shown in Figure 2(a), we detected a significant $(P=0.022)$ difference in serum ALT activities between carriers of different PNPLA3 genotypes. In particular, homozygous risk allele carriers (i.e., with the genotype PNPLA3 p.148Met/Met) presented with 


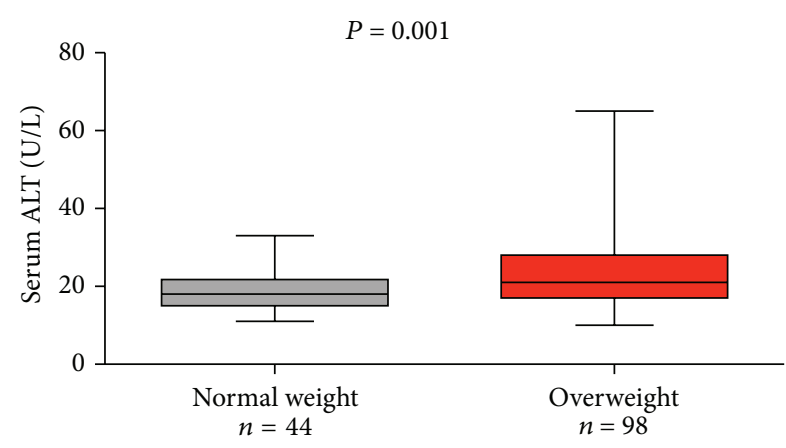

(a)

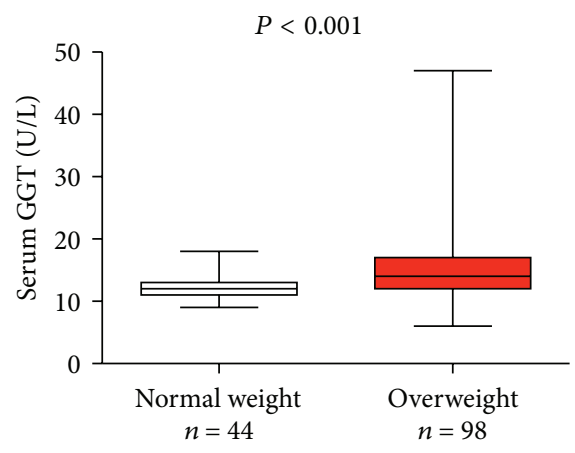

(b)

FIGURE 1: Obese children present with increased serum ALT and GGT activities. Serum ALT (a) and GGT (b) activities differed significantly $(P=0.001$ and $P<0.001$, resp.) between obese and normal weight children. We did not detect any significant differences between both groups with respect to the AST activities $(P>0.05)$.

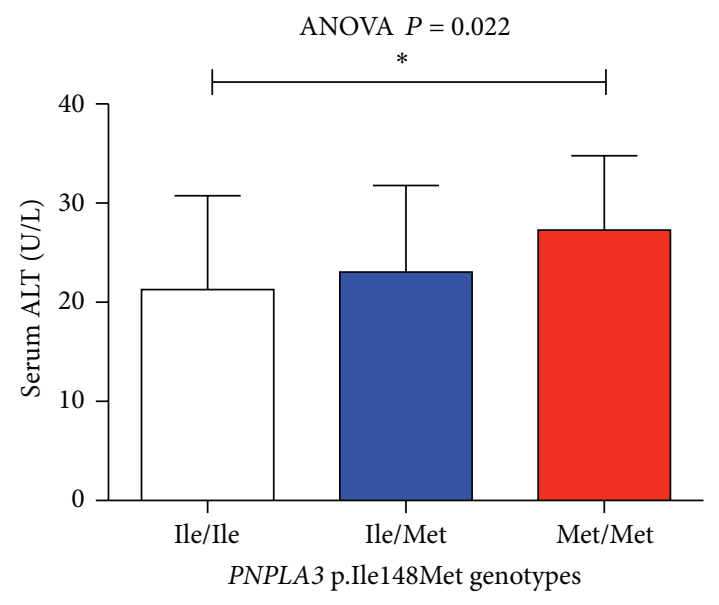

(a)

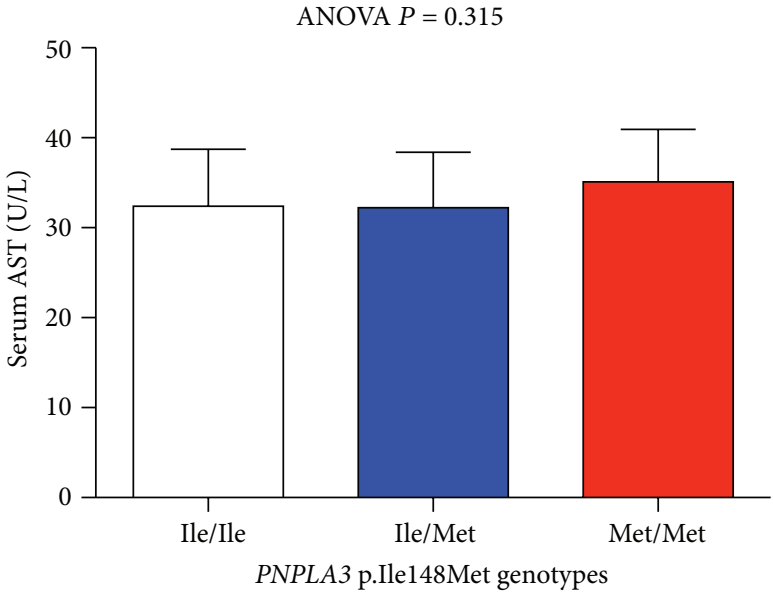

(b)

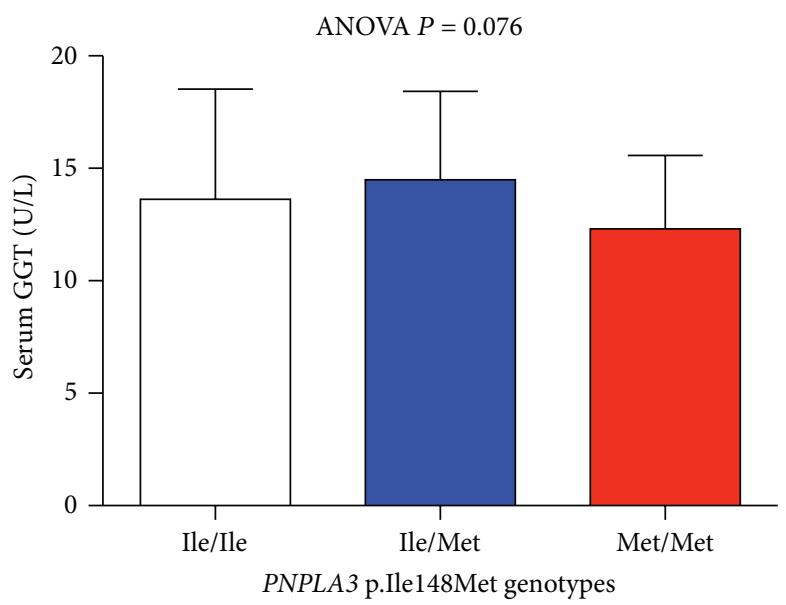

(c)

FIgURE 2: The prosteatotic PNPLA3 variant p.Ile148Met is associated with higher serum ALT activity at young age. Serum ALT activities differ significantly (ANOVA $P=0.022$ ) between carriers of different PNPLA3 genotypes. Especially children carrying the PNPLA3 p.148Met/Met variant present with significantly $\left({ }^{*} P=0.009\right)$ higher ALT as compared to the carriers of the common variant PNPLA3 p.148Ile/Ile (a). Presence of this variant was not associated with other liver function tests ((b) and (c)). 
significantly $(P=0.009)$ higher median serum ALT (27 IU, range 17-40 IU) as compared to carriers of the genotype PNPLA3 p.148Ile/Ile (median 19 IU, range 10-60 IU).

In separate analyses in normal weight and overweight children the association remained significant in the nonoverweight cohort (ANOVA $P=0.016$ ): Carriers of the PNPLA3 genotype p.148Met/Met $(N=5)$ presented with higher serum ALT activities (median 27, range 19-33) than carriers of the genotype p.148Ile/Ile $(N=29$, median 16 , range $12-32$, $P=0.005)$ and to individuals with the genotype PNPLA3 p.148Ile/Met $(N=10$, median 17.5 , range $11-28, P=0.016)$. In univariate regression analyses, both the PNPLA3 genotype and the BMI were associated with increased ALT $(P=0.049$ and $P<0.001$, resp.). In the multivariate analysis we detected a strong association between increased ALT and BMI $(P<$ $0.001)$ and a trend for the association with variant PNPLA3 $(P=0.056)$. To investigate whether the PNPLA3 polymorphism has different effects on liver parameters in different age groups we stratified the cohort into two subhorts: aged 5-6 years $(n=41)$ and aged $7-9$ years $(n=101)$. We detected a significant association between higher ALT activities and the PNPLA3 risk variant in the younger $(P=0.017)$ but not in the older children. However, the latter cohort comprised significantly $(P=0.04)$ more obese individuals. We did not detect any association between the studied variant and serum activities of AST or GGT (Figures 2(b) and 2(c)). Moreover, we detected significant differences in fasting serum glucose levels and IR-HOMA between carriers of the PNPLA3 genotypes (Table 2, ANOVA $P=0.01$ and ANOVA $P=0.02$, resp.), which were the highest in carriers of the p.148Met/Ile genotype. The variant was however not associated with other metabolic (Table 2) or anthropometric traits in the studied cohort (all $P>0.05)$.

\section{Discussion}

This study investigates the impact of the common PNPLA3 variant p.Ile148Met on liver health in a cohort of German children and describes an association of the risk allele p.148Met with liver injury as indicated by higher serum ALT activities. We identify an at risk population that is likely to develop liver injury rather than a population of sick children. Indeed, only a small number of individuals in our cohort have already developed fatty liver, and increased serum ALT activities in most carriers of the risk variant stay within what is considered the normal range (Figure 2(a)). However, extrapolating from older cohorts [14-16], it is obvious that liver injury and subsequent development of clinical symptoms are likely to follow unless a change in lifestyle is pursued. Indeed, previous studies (e.g., [17]) showed that increased ALT values within the normal range are clinically relevant; moreover, it was previously postulated that the currently accepted ALT serum thresholds are set too high to detect hepatopathies in pediatric patients [18].

Prior results from studies on the impact of PNPLA3 p.Ile148Met genotypes in pediatric cohorts were variable, probably due to differences in the groups tested. Valenti et al. [7] sampled 149 children and adolescents (mean age of 10.2 years) and observed a striking association of the PNPLA3
p.148Met allele with NASH and fibrosis, but not with serum AST or ALT. Giudice et al. [8] tested 1058 patients aged between 2 and 16 years (mean age 10.6 years) and found an association of the PNPLA3 prosteatotic allele with higher serum AST and ALT activities. Particularly pronounced in the subgroup with a high level of abdominal fat (waist-toheight ratio $>0.62$ ). These data were in agreement with findings by Romeo et al. [6] in 475 obese children and adolescents (mean age 10.3 years) who showed significantly increased serum ALT and AST values for carriers of the p.148Met allele. Interestingly, a recent analysis of children aged 6-8 years showed an association between the PNPLA3 variant and increased liver tests in obese but not in normal weight children [19]. This association even increased in the 2-year follow-up [19]. In our cohort, in contrast, the PNPLA3 risk genotype was associated with serum ALT in normal weight children. Although overweight children carrying the p.148Met/Met variant presented with increased ALT activities, the difference in comparison to carriers of the p.148Ile/Ile genotype was not significant $(P=0.068)$. The apparent discrepancies between these observations might be related, for example, to the presence of different nongenetic triggers of liver injury (e.g., diet), which might modulate the interaction between genetic predisposition, BMI, and liver disease. Of note, our results in normal weight children are in line with a previous Mexican study analyzing a cohort of 1037 children aged 6-12 years [20]. This study [20] demonstrated that the PNPLA3 risk variant has a stronger effect on serum ALT in normal weight children than in overweight and obese children. In our study we included children with an average age of 7 years; hence we depict a very early association between the genotype and the presence of increased serum ALT activities. On the other hand, the PNPLA3 risk allele had a lower effect on serum transaminases as compared to increased body weight.

A meta-analysis of 16 studies confirmed the impact of the PNPLA3 on liver fat content and the severity of NAFLD as measured by necroinflammatory scores and fibrosis stages [21]. In our cohort we did not detect any significant association between the PNPLA3 variant and the presence of fatty liver. This might be related to the fact that only $13 \%$ of participating children had fatty liver. Our results demonstrate that hepatic injury, as reflected by increased serum transaminases, is present in young children carrying the rare PNPLA3 variant already before fatty liver can be detected. The authors of the previous studies (reviewed in detail in [14]) hypothesized that PNPLA3 may possess both lipase and triglyceride synthase activities. The analysis [22] of the Pnpla3 p.Ile148Met knockin mice demonstrated that the prosteatotic allele p.148Met may lead to an increased expression of PNPLA3 on lipid droplets under a high-sucrose diet. Since it is not entirely clear yet how the PNPLA3 risk variant impacts lipase function and facilitates the cell damage evidenced by increased ALT levels, it is essential to study the resulting clinical phenotype in great detail and under various circumstances, that is, in different age groups. Interestingly, we detected significant differences in glucose levels and IR-HOMA among carriers of the PNPLA3 genotypes (Table 2). These were highest among children with the p.148Ile/Met genotype, which confers 
TABLE 2: Metabolic traits in relation to the PNPLA3 p.Ile148Met genotypes.

\begin{tabular}{|c|c|c|c|c|}
\hline \multirow{2}{*}{ Feature } & \multicolumn{3}{|c|}{ PNPLA3 p.Ile148Met genotype } & \multirow{2}{*}{$P$ value } \\
\hline & Ile/Ile $(n=75)$ & Ile/Met $(n=57)$ & Met/Met $(n=10)$ & \\
\hline Triglycerides (mg/dL) & $70.0(25.0-294.0)$ & $69.0(35.0-231.0)$ & $57.5(41.0-92.0)$ & 0.39 \\
\hline HDL cholesterol (mg/dL) & $54.0(26.0-71.0)$ & $52.0(21.0-77.0)$ & $50.5(38.0-64.0)$ & 0.55 \\
\hline LDL cholesterol (mg/dL) & $108.0(63.0-155.0)$ & $114.0(62.0-166.0)$ & $99.5(93.0-124.0)$ & 0.09 \\
\hline Total cholesterol (mg/dL) & $176.0(113.0-230.0)$ & $185.0(121.0-245.0)$ & $164.5(145.0-187.0)$ & 0.06 \\
\hline Glucose $(\mathrm{mg} / \mathrm{dL})$ & $84.5(72.0-103.0)$ & $87.0(63.0-122.0)$ & $84.5(75.0-94.0)$ & 0.01 \\
\hline Insulin $(\mu \mathrm{lU} / \mathrm{mL})$ & $10.0(4.1-38.8)$ & $11.0(1.9-33.7)$ & $8.4(2.8-17.0)$ & 0.06 \\
\hline IR-HOMA & $2.0(1.0-9.2)$ & $2.6(0.5-7.7)$ & $1.6(0.6-3.6)$ & 0.02 \\
\hline
\end{tabular}

Ile: isoleucine; IR-HOMA: insulin resistance homeostasis model assessment; Met: methionine; p: protein (amino acid number); PNPLA3: adiponutrin. Values are given as medians and ranges.

an intermediate NAFLD-risk. The impact of the PNPLA3 genotype on insulin resistance is controversial. Although in most published studies no association between the PNPLA3 variant and insulin resistance or glucose levels was detected [14], a few studies reported significant effects of the PNPLA3 variant on metabolic status [23-26]. The weaknesses of our study include a relatively small sample size and a lack of liver biopsy or MRI as measurements of hepatic steatosis. MRI would also allow analysis of body fat composition and investigate its interactions between the PNPLA3 genotype, body fat distribution, and liver injury.

\section{Conclusion}

Our data from a relatively small German pediatric cohort suggest that young children bearing the homozygous risk allele of PNPLA3 p.Ile148Met suffer from liver damage at an early age. We did not detect any association between the studied variant and the presence of other traits that are commonly linked to increased hepatic fat accumulation, apart from increased glucose levels and IR-HOMA in individuals with the Ile/Met variant. Hence, at risk pediatric patients may be easily missed when increased BMI is used as a cut-off mark for screening individuals at risk of developing NAFLD. Thus, screening of children for the PNPLA3 risk allele regardless of their body weight may be useful in regards to an early determination of "at-risk" children. While we are aware of the risk of stigmatising children by subjecting them to genotyping and allocating them to an "at-risk" group, it is our view that the benefit derived from informing parents in a confidential and thorough manner will outweigh any such risk. Further studies are urgently needed to develop tailored genotype-guided strategies for the prevention of NAFLD and its consequences in children.

\section{List of Abbreviations}

ANOVA: Analysis of variance

BMI-SDS: Body mass index-standard deviation score

HWE: Hardy-Weinberg equilibrium

IR-HOMA: Insulin resistance homeostasis model assessment

NAFLD: Nonalcoholic fatty liver disease
NASH: Nonalcoholic steatohepatitis

PNPLA3: Patatin-like phospholipase domain containing 3 (adiponutrin).

\section{Conflict of Interests}

The authors declare that they have no conflict of interests.

\section{Acknowledgments}

This work was supported by HOMFOR program of Saarland University to Marcin Krawczyk and the German Ministery for Research and Education (BMBF, FKZ:01EA1305) to Ina Bergheim.

\section{References}

[1] M. Blachier, H. Leleu, M. Peck-Radosavljevic, D. C. Valla, and F. Roudot-Thoraval, "The burden of liver disease in Europe: a review of available epidemiological data," Journal of Hepatology, vol. 58, no. 3, pp. 593-608, 2013.

[2] H. M. Patton, C. Sirlin, C. Behling, M. Middleton, J. B. Schwimmer, and J. E. Lavine, "Pediatric nonalcoholic fatty liver disease: a critical appraisal of current data and implications for future research," Journal of Pediatric Gastroenterology and Nutrition, vol. 43, no. 4, pp. 413-427, 2006.

[3] A. Wree, L. Broderick, A. Canbay, H. M. Hoffman, and A. E. Feldstein, "From NAFLD to NASH to cirrhosis-new insights into disease mechanisms," Nature Reviews Gastroenterology o Hepatology, vol. 10, no. 11, pp. 627-636, 2013.

[4] V. Giorgio, F. Prono, F. Graziano, and V. Nobili, "Pediatric non alcoholic fatty liver disease: old and new concepts on development, progression, metabolic insight and potential treatment targets," BMC Pediatrics, vol. 13, no. 1, article 40, 2013.

[5] S. Romeo, J. Kozlitina, C. Xing et al., "Genetic variation in PNPLA3 confers susceptibility to nonalcoholic fatty liver disease," Nature Genetics, vol. 40, no. 12, pp. 1461-1465, 2008.

[6] S. Romeo, F. Sentinelli, V. M. Cambuli et al., "The 148M allele of the PNPLA3 gene is associated with indices of liver damage early in life," Journal of Hepatology, vol. 53, no. 2, pp. 335-338, 2010.

[7] L. Valenti, A. Alisi, E. Galmozzi et al., "I148M patatin-like phospholipase domain-containing 3 gene variant and severity of pediatric nonalcoholic fatty liver disease," Hepatology, vol. 52, no. 4, pp. 1274-1280, 2010. 
[8] E. M. Giudice, A. Grandone, G. Cirillo et al., "The association of PNPLA3 variants with liver enzymes in childhood obesity is driven by the interaction with abdominal fat," PLOS ONE, vol. 6, no. 11, Article ID e27933, 2011.

[9] I. B. Maier, Y. Özel, S. Wagnerberger, S. C. Bischoff, and I. Bergheim, "Dietary pattern and leisure time activity of overweight and normal weight children in Germany: sex-specific differences," Nutrition Journal, vol. 12, no. 1, article 14, 2013.

[10] K. Kromeyer-Hauschild, M. Wabitsch, D. Kunze et al., "Percentiles of body mass index in children and adolescents evaluated from different regional German studies," Monatsschrift fur Kinderheilkunde, vol. 149, no. 8, pp. 807-818, 2001.

[11] A. E. Joseph, S. H. Saverymuttu, S. Al-Sam, M. G. Cook, and J. D. Maxwell, "Comparison of liver histology with ultrasonography in assessing diffuse parenchymal liver disease," Clinical Radiology, vol. 43, no. 1, pp. 26-31, 1991.

[12] J. P. Hosker, D. R. Matthews, A. S. Rudenski et al., "Continuous infusion of glucose with model assessment: measurement of insulin resistance and beta-cell function in man," Diabetologia, vol. 28, no. 7, pp. 401-411, 1985.

[13] M. Krawczyk, F. Grünhage, V. Zimmer, and F. Lammert, "Variant adiponutrin (PNPLA3) represents a common fibrosis risk gene: non-invasive elastography-based study in chronic liver disease," Journal of Hepatology, vol. 55, no. 2, pp. 299-306, 2011.

[14] M. Krawczyk, P. Portincasa, and F. Lammert, "PNPLA3-associated steatohepatitis: toward a gene-based classification of fatty liver disease," Seminars in Liver Disease, vol. 33, no. 4, pp. 369379, 2013.

[15] Y. L. Liu, G. L. Patman, J. B. S. Leathart et al., "Carriage of the PNPLA3 rs738409 C > G polymorphism confers an increased risk of non-alcoholic fatty liver disease associated hepatocellular carcinoma," Journal of Hepatology, vol. 61, no. 1, pp. 75-81, 2014.

[16] E. Trépo, P. Nahon, G. Bontempi et al., "Association between the PNPLA3 (rs738409 C > G) variant and hepatocellular carcinoma: evidence from a meta-analysis of individual participant data," Hepatology, vol. 59, no. 6, pp. 2170-2177, 2014.

[17] H. C. Kim, C. M. Nam, S. H. Jee, K. H. Han, D. K. Oh, and I. Suh, "Normal serum aminotransferase concentration and risk of mortality from liver diseases: prospective cohort study," British Medical Journal, vol. 328, no. 7446, pp. 983-986, 2004.

[18] J. B. Schwimmer, W. Dunn, G. J. Norman et al., "SAFETY study: alanine aminotransferase cutoff values are set too high for reliable detection of pediatric chronic liver disease," Gastroenterology, vol. 138, no. 4, pp. 1357.e2-1364.e2, 2010.

[19] A. Viitasalo, J. Pihlajamaki, V. Lindi et al., "Associations of I148M variant in PNPLA3 gene with plasma ALT levels during 2-year follow-up in normal weight and overweight children: the PANIC study," Pediatric Obesity, vol. 10, no. 2, pp. 84-90, 2015.

[20] E. Larrieta-Carrasco, P. León-Mimila, T. Villarreal-Molina et al., "Association of the I148M/PNPLA3 variant with elevated alanine transaminase levels in normal-weight and overweight/ obese Mexican children," Gene, vol. 520, no. 2, pp. 185-188, 2013.

[21] S. Sookoian and C. J. Pirola, "Meta-analysis of the influence of I148M variant of patatin-like phospholipase domain containing 3 gene (PNPLA3) on the susceptibility and histological severity of nonalcoholic fatty liver disease," Hepatology, vol. 53, no. 6, pp. 1883-1894, 2011.

[22] E. Smagris, S. BasuRay, J. Li et al., "Pnpla3I148M knockin mice accumulate PNPLA3 on lipid droplets and develop hepatic steatosis," Hepatology, vol. 61, no. 1, pp. 108-118, 2015.
[23] M. Krawczyk, F. Gruenhage, M. Mahler, S. Tirziu, M. Acalovschi, and F. Lammert, "The common adiponutrin variant p.I148m does not confer gallstone risk but affects fasting glucose and triglyceride levels," Journal of Physiology and Pharmacology, vol. 62, no. 3, pp. 369-375, 2011.

[24] C. N. A. Palmer, C. Maglio, C. Pirazzi et al., "Paradoxical lower serum triglyceride levels and higher type 2 diabetes mellitus susceptibility in obese individuals with the PNPLA3 148M variant," PLoS ONE, vol. 7, no. 6, Article ID e39362, 2012.

[25] K. Rembeck, C. Maglio, M. Lagging et al., "PNPLA 3 I148M genetic variant associates with insulin resistance and baseline viral load in HCV genotype 2 but not in genotype 3 infection," BMC Medical Genetics, vol. 13, article 82, 2012.

[26] C.-W. Wang, H.-Y. Lin, S.-J. Shin et al., "The PNPLA3 I148M polymorphism is associated with insulin resistance and nonalcoholic fatty liver disease in a normoglycaemic population," Liver International, vol. 31, no. 9, pp. 1326-1331, 2011. 


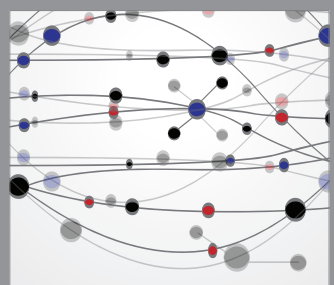

The Scientific World Journal
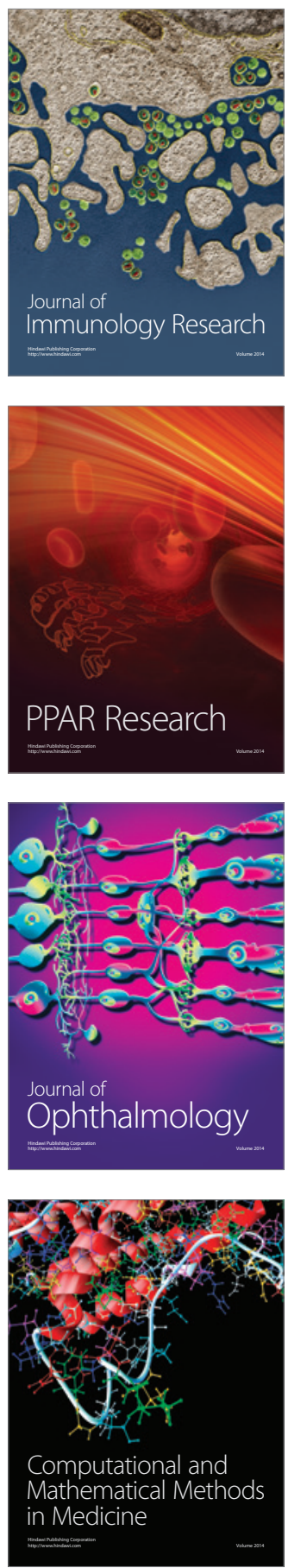

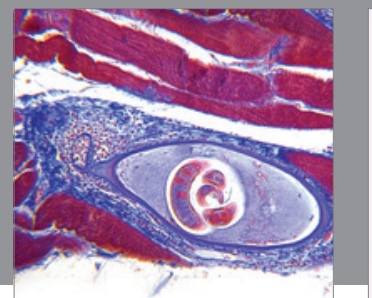

Gastroenterology

Research and Practice
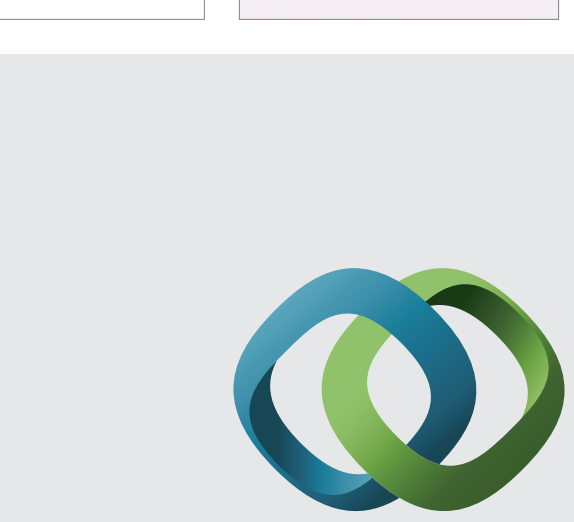

\section{Hindawi}

Submit your manuscripts at

http://www.hindawi.com
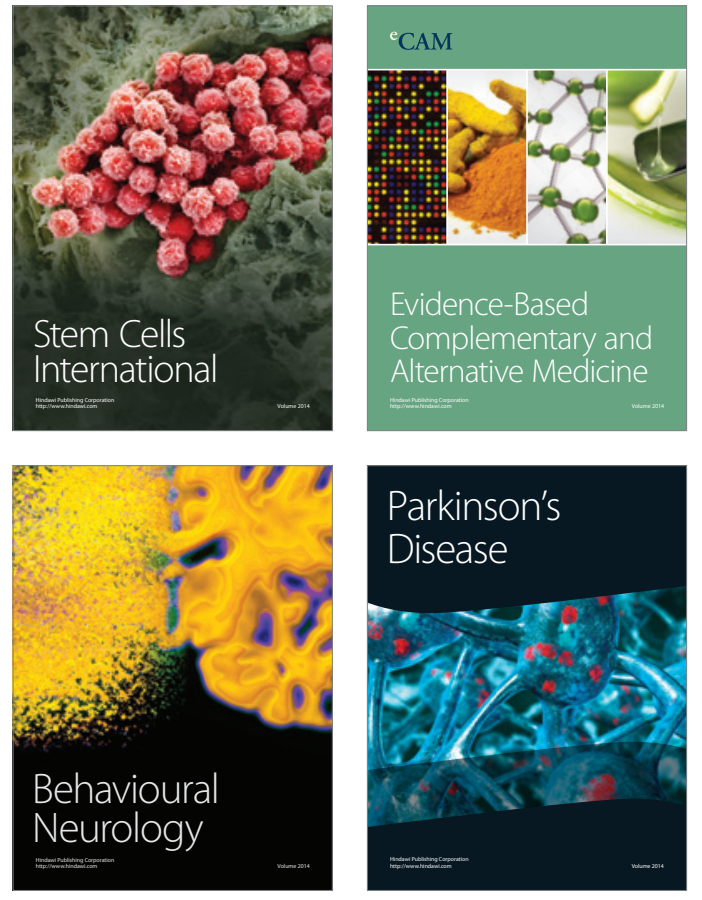
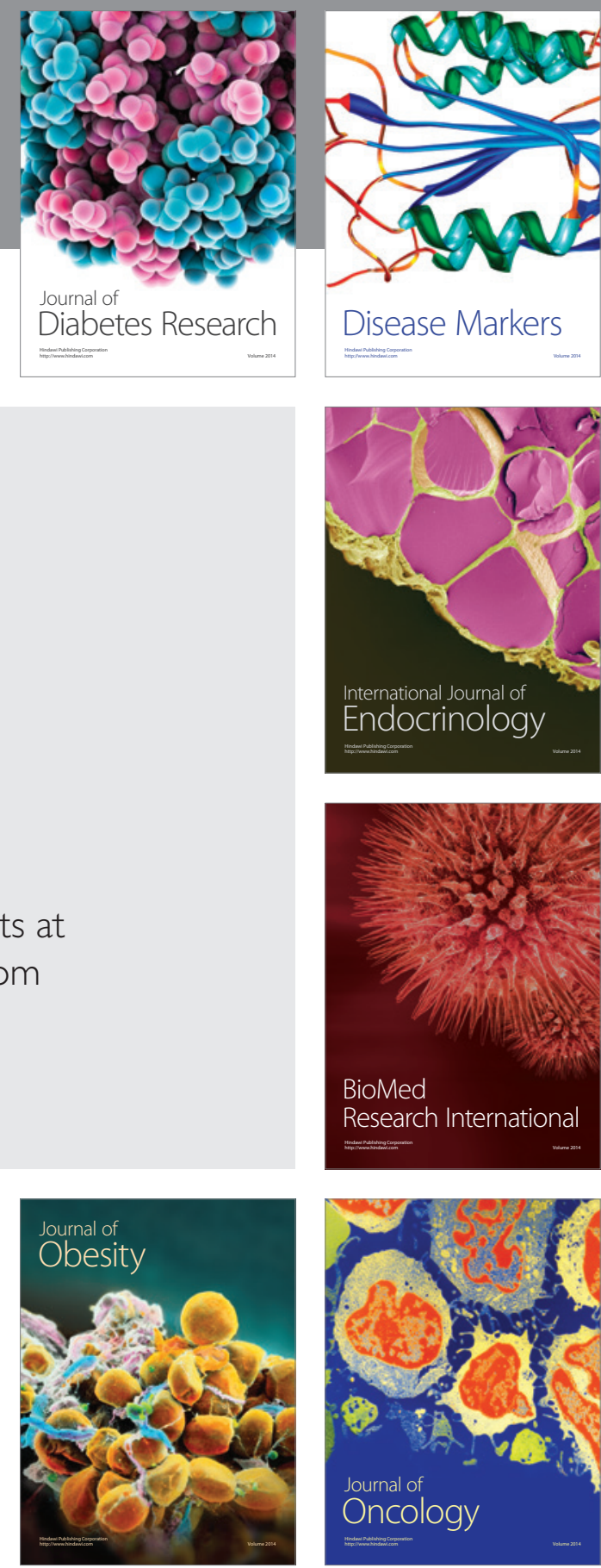

Disease Markers
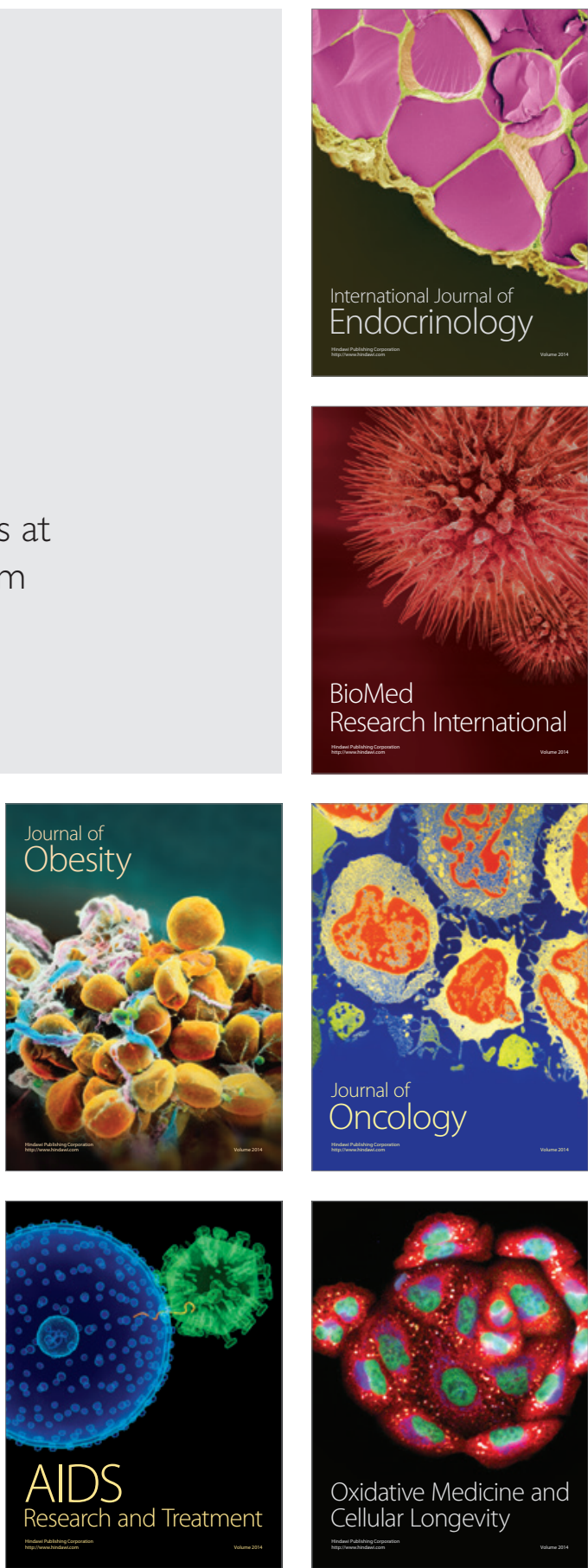